\title{
Erratum to: Quantitative Assessment of Turbulence and Flow Eccentricity in an Aortic Coarctation: Impact of Virtual Interventions
}

\author{
Magnus Andersson, ${ }^{1,5}$ Jonas Lantz, ${ }^{2,5}$ Tino Ebbers, ${ }^{2,3,4,5}$ and Matts Karlsson ${ }^{1,4,5}$ \\ ${ }^{1}$ Department of Management and Engineering (IEI), Linköping University, 581 83Linköping, Sweden; ${ }^{2}$ Department of Science \\ and Technology, Linköping University, Linköping, Sweden; ${ }^{3}$ Department of Medical and Health Sciences, Linköping \\ University, Linköping, Sweden; ${ }^{4}$ Center for Medical Image Science and Visualization (CMIV), Linköping University, Linköping, \\ Sweden; and ${ }^{5}$ Swedish e-Science Research Center (SeRC), Stockholm, Sweden
}

(Published online 14 September 2015)

\begin{abstract}
Turbulence and flow eccentricity can be measured by magnetic resonance imaging (MRI) and may play an important role in the pathogenesis of numerous cardiovascular diseases. In the present study, we propose quantitative techniques to assess turbulent kinetic energy (TKE) and flow eccentricity that could assist in the evaluation and treatment of stenotic severities. These hemodynamic parameters were studied in a pre-treated aortic coarctation (CoA) and after several virtual interventions using computational fluid dynamics (CFD), to demonstrate the effect of different dilatation options on the flow field. Patient-specific geometry and flow conditions were derived from MRI data. The unsteady pulsatile flow was resolved by large eddy simulation (LES) including non-Newtonian blood rheology. Results showed an inverse asymptotic relationship between the total amount of TKE and degree of dilatation of the stenosis, where the pre-stenotic hypoplastic segment may limit the possible improvement by treating the CoA alone. Spatiotemporal maps of TKE and flow eccentricity could be linked to the characteristics of the post-stenotic jet, showing a versatile response between the $\mathrm{CoA}$ dilatations. By including these flow markers into a combined MRI-CFD intervention framework, CoA therapy has not only the possibility to produce predictions via simulation, but can also be validated pre- and immediate post treatment, as well as during followup studies.
\end{abstract}

Keywords-Computational fluid dynamics, Large eddy simulation, Turbulent kinetic energy, Flow displacement, NonNewtonian, Carreau, Virtual treatment, Magnetic resonance imaging.

Address correspondence to Magnus Andersson, Department of Management and Engineering (IEI), Linköping University, 581 83Linköping, Sweden. Electronic mail: magnus.andersson@liu.se

The online version of the original article can be found under doi: 10.1007/s13239-015-0218-x.

\section{ERRATUM TO: CARDIOVASCULAR ENGINEERINGAND TECHNOLOGY (2015) 6(3):281-293 DOI 10.1007/S13239-015-0218-X}

This erratum originates from a fault in the boundary conditions of the computational models, resulting in elevated flow conditions through the descending aorta with respect to the measured patient-specific data. This was not in line with the numerical setup addressed in the paper. Hence, this matter was adjusted to the appropriate physiological state and each case re-simulated accordingly. In spite of this revision all conclusions of the paper are unaltered.

\section{INTRODUCTION}

Blood flow distal to abnormal morphologies such as stenoses, aneurysms, abnormal aortic valves, unfavorable branching, kinks, lesions and other irregularities of the vessel wall is often dominated by eccentric and turbulent hemodynamics. Turbulent flow results in reduced blood transport efficiency as turbulent kinetic energy (TKE) is drained from the energy in the main flow. ${ }^{51}$ In contrast to well-structured laminar flows, turbulent flow has been associated to a wide spectrum of diseases e.g., post-stenotic dilatation and aneurysms formation, progression of atherosclerosis, blood damage, induced endothelial cell turnover and thrombus formation. ${ }^{41}$ Also outflow jet eccentricity has been associated with the aortic dilation and aneurysm formation, e.g., patients with bicuspid aortic valve (BAV) disease where significant flow displacement and jet-towall impingement could be observed compared to the general population. . $^{3,14,17,50}$

Coarctation of the aorta (CoA) is a congenital cardiovascular malformation characterized by an iso- 
lated narrowing of the vessel lumen accompanied with jet-like and turbulent flow characteristics, leading to upper-body hypertension as a result of the increased trans-stenotic pressure drop. Despite recent advancement in intervention procedures, premature reduced life expectancy is frequently observed and linked to long-term cardiovascular complications. Late morbidity of post-coarctectomy can be recoarctation (reCoA), aortic aneurysm formation or aortic dissection, coexisting aortic valve abnormalities, endocarditis, premature coronary atherosclerosis, cerebrovascular diseases and persistent systemic hypertension at rest and exercise, $,, 52,56$ even without stents or mild reCoA. ${ }^{21}$ The mechanisms behind these cardiovascular events are not fully understood yet, but are believed to partly stem from altered abnormal hemodynamic features triggered by a non-optimal aortic morphology, ${ }^{41,44}$ and/or persistent and continued biomechanical degradation of the arterial wall. ${ }^{21,38,43}$

Today, aortic coarctations are corrected by surgical treatment or by catheter interventions, ${ }^{47}$ where the main objective is to efficiently restore systemic blood flow and minimize the trans-coarctation pressure gradient. However, in spite of satisfactory repairs, recovering the pressure gradient to an acceptable limit, late complications are not uncommon. To address these problematic events, research have turned to computational fluid dynamics (CFD) to simulate the outcome of different CoA interventions, ${ }^{10-12,29-31,38}$ where pressure drop and wall shear stress (WSS) are some of the evaluated parameters. However, a downside with most of these flow descriptors is that they can only be acquired with reasonable accuracy by invasive methods or via CFD.

Non-invasive hemodynamic assessment in CoAs has for long been limited to the estimation of the peak velocity in the jet, which sometimes is used clinically to estimate the pressure drop using the simplified Bernoulli equation. But recently, turbulent kinetic energy and flow eccentricity, obtained non-invasively using 4D flow MRI, ${ }^{24,36}$ have been introduced as hemodynamic markers. ${ }^{16,25,50,60}$ MRI-based TKE levels have showed to be closely linked to pressure loss in stenotic flows, ${ }^{5,15}$ and to be in good agreement with scale-resolving CFD simulations. ${ }^{2,32}$ Furthermore, elevated turbulence has shown to induce abnormal WSS ${ }^{18,19,33}$ and increase local flow strain-rates significantly. ${ }^{18}$ Also eccentric flows have been related to increased growth rate of aortic dilations, ${ }^{25}$ elevated and complex WSS patterns at the vicinity of jet-impingement, ${ }^{3,17,55}$ favor turbulence production ${ }^{28,53}$ and produce large zones of vortex formation and flow recirculation, regions known to promote platelet thrombus formation. ${ }^{26}$

While non-invasive assessment of TKE and eccentric flow has led to several interesting findings, they have not been used extensively in CFD studies of
CoAs. In a study by Lantz et al., ${ }^{32}$ CFD was used to review the turbulence distal to a CoA before and after catheter intervention, revealing a substantial decrease in peak and volume integrated TKE as a result of the intervention. However, the same study also showed a noticeable amount of residual turbulence post treatment, suggesting that the CoA morphology was not restored sufficiently and that turbulence quantification could act as a marker to assess this severity. More research is necessary to understand the role of these markers in the development and treatment of CoAs.

In this work, we propose novel quantitative techniques to assess turbulent kinetic energy and flow eccentricity, which may offer additional information for improving the long-term outcome of CoA therapy. These parameters were studied using CFD in a patientspecific CoA-model before treatment and after a range of virtual interventions, with the objective to investigate the effect of different dilatation options on the flow field. As both TKE and flow eccentricity can be measured non-invasively by MRI and computed by CFD, an integrated intervention framework can not only be used to produce predictions via simulation, but also to validate the CoA severity pre- and immediate post treatment, as well as during follow-up studies.

\section{METHOD}

The pre-treated computational model (Baseline) was derived from MRI data (Fig. 1) and consists of the ascending aorta (AAo), brachiocephalic artery (BCA), left common carotid artery (LCCA), hypoplastic aortic arc (Kink), coarctation of the aorta (CoA), descending aorta (DAo) and parts of the thoracic aorta (TAo). This section provides a general overview of the method; more details are given in the supplementary material (Online Resource 1).

(a)

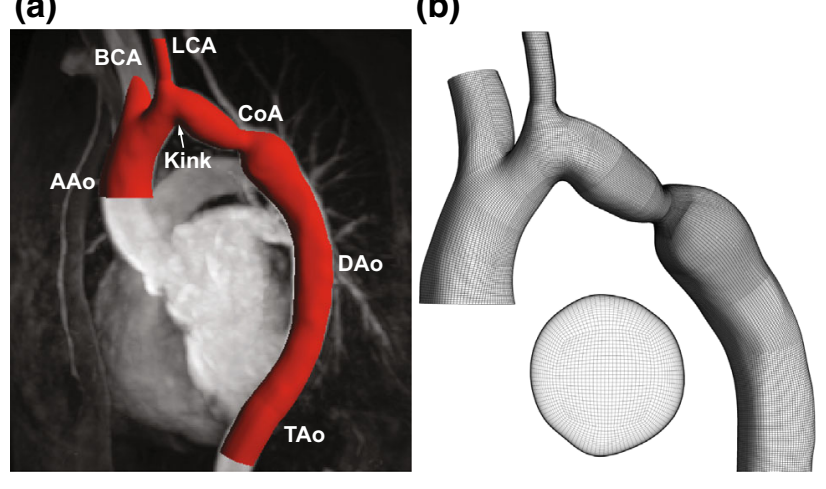

FIGURE 1. (a) Maximum Intensity Projection (MIP) image of the MRI data with imposed segmented geometry (red). (b) Computational mesh. 


\section{MRI Acquisition and Segmentation}

The MRI measurements were performed on a 63-year old female with a reCoA. Proper consent and approval were acquired from the patient and local ethics committee. The central blood vessels were imaged by a contrast-enhanced MR angiography, and reconstructed to spatial resolution of $0.88 \times 0.88 \times 4 \mathrm{~mm}^{3}$. Supracoronary flow was obtained from unidirectional velocity dataset using 2D through-plane PC-MRI, reconstructed to 40 time-frames per cardiac cycle with an in-plane spatial resolution of $1.56 \times 1.56 \mathrm{~mm}^{2}$. Both the angiogram and flow measurements were obtained within an expiration breath-hold. All image analysis was performed by the freely available software Segment ${ }^{23}$ ( http://segment.heiberg.se). The geometry was obtained through a $3 \mathrm{D}$ level set segmentation technique, providing a stereolithography representation of the lumen boundary (Fig. 1a). The degree of stenosis was 65\% based on the cross-sectional area.

\section{Intervention Simulation}

Prior to the virtual intervention the pre-treated CoA revealed a strong jet exiting the throat of the stenosis, promoting both severe turbulent as well as eccentric flow conditions distally. With these observations in hand, six virtual interventions were proposed, each dilated to a different degree in order to make the coarctations less eccentric with respect to the nearby morphology. The dilation simulations of the CoA were simulated using ANSYS Mechanical 14.0 over an isolated segment (Fig. 2). The segmented surface was meshed using shell elements with uniform wall thickness and linear elastic wall properties. To enhance the flexibility of the virtual dilatation, the model was divided into several sub-sections where internal pressure profiles and supports could be tuned in order obtain the desirable load conditions, for details see Online Resource. The post-treated cases are categorized from smallest (Case 1) to the largest stenotic cross-sectional area increase (Case 6), also denoted as C1-C6 (Fig. 2). To assess a more standard way of performing an angioplasty two concentric dilations was acquired (C3 and $\mathrm{C6}$ ), which were more isotropically deformed around the circumference. In relation to the pre-treated coarctation (BL), three stages of eccentric inner curvature dilatation $(\mathrm{C} 2, \mathrm{C} 4$ and $\mathrm{C} 5)$ and one case of outer curvature dilation $(\mathrm{C} 1)$ were simulated, all with the potential to restoring the flow differently.

\section{Flow Model}

For transitional and turbulent blood flow different numerical methods can be adopted in order to model and/or resolve the wide ranges of flow structures. ${ }^{6}$ The overall poor performance of Reynolds Average Navier-Stokes (RANS) based models and the inapplicability of using Direct Numerical Simulation (DNS) schemes for realistic high Reynolds number vascular flows are well accepted. ${ }^{32,39,48,49,59}$ In between DNS and RANS modeling, Large Eddy Simulation (LES) on the other hand only resolves the largest energycontaining scales whereas the remaining unresolved part of the flow is handled by a subgrid-scale (SGS) model. LES have been performed in a number of idealized stenotic flow studies, ${ }^{19,39,45}$ showing excellent agreement with experimental data. More recently, LES have also been employed for investigating the WSS and low-density lipoproteins interaction with local flow disturbances in a subject-specific aorta. ${ }^{33,34}$ In this study blood flow was resolved by LES using the walladapted local eddy viscosity (WALE) SGS model. ${ }^{34,42}$

In pulsatile turbulent flow the non-linearity of blood rheology can have impact on flow characteristics and TKE predictions. ${ }^{37,40}$ For this study the fluid was
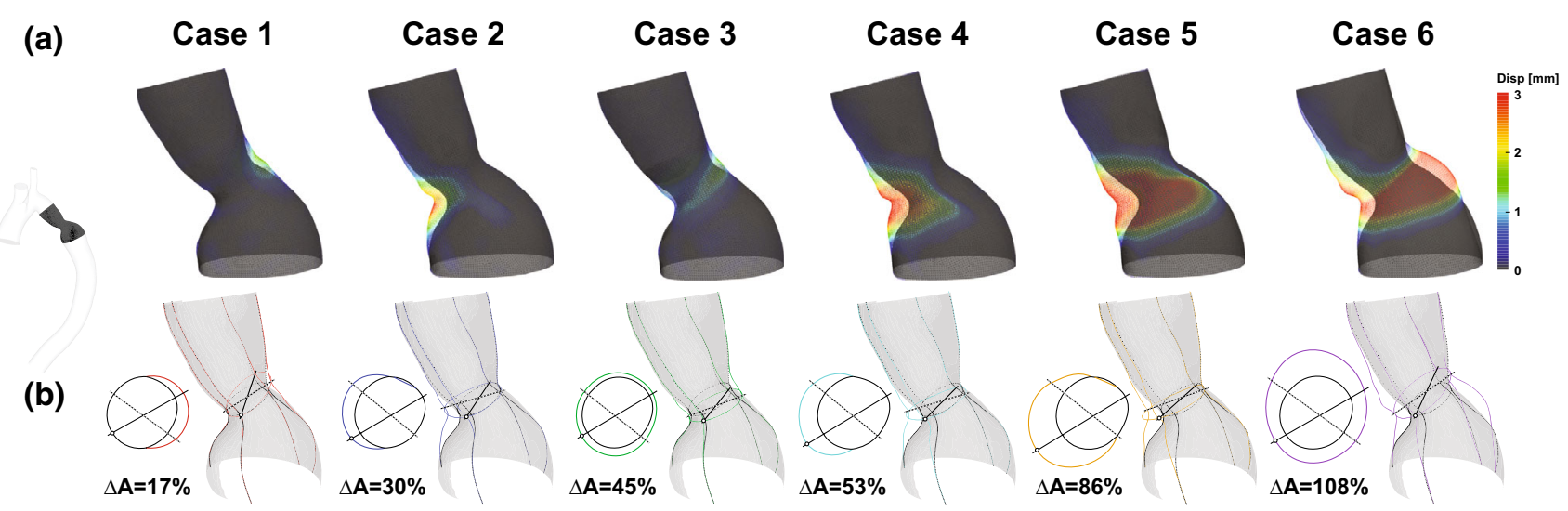

FIGURE 2. Segments of the post-intervention configurations showing the deformation with respect to the pre-treated topology, also indicated to the left. (a) Deformation wireframe with color-coded displacement. (b) The degree of dilatation, categorized from the smallest (Case 1) to the largest (Case 6) stenotic cross-sectional area. 
assumed to be non-Newtonian, modelled by the fourparameter Carreau model ${ }^{6}$ and fitted against in vitro data from Cho and Kensey. ${ }^{8}$

The numerical simulation was performed in ANSYS CFX 15.0. Patient-specific volumetric flow rates were assigned at the AAo, corresponding to a cardiac output of $51 \mathrm{~min}^{-1}$. The outflow distribution through the BCA and the LCCA were determined by a square law governed by the flow and diameter relation of the AAo and the first branching vessels. ${ }^{61}$ The vessel wall was assumed to be rigid with an applied non-slip condition. A zero static pressure boundary condition was assigned to the outlet of the TAo. After intervention a worst case scenario of maintained flow rate was assumed.

The hexahedral meshes (Fig. 1b) were constructed in ANSYS ICEM CFD 14.0, and resulted in 3 million cells (MC). The non-dimensional wall distance y-plus was below unity and a low cell growth rate was used to ensure that the near-wall boundary layer was well resolved. As the SGS model explicitly is a function of the mesh, there is no conventional way of validating meshindependency. In this work proper LES grid resolution was checked by two-point correlations in several streamwise and radial directions downstream the coarctation during steady peak flow conditions. Results showed that on average 8 mesh cells was used to resolve the largest flow structures, which can be considered to be sufficient. ${ }^{13}$ To assess the sensitivity to grid refinement, an additional $2.5 \mathrm{MC}$ were added to the turbulent regions. The volume integrated TKE showed only a minor root-mean-square deviation $($ RMSD) over the cardiac cycle $(\mathrm{RMSD}=0.1 \mathrm{~mJ})$ and the total TKE difference was $3 \%$ compared to the 3 MC mesh, indicating an acceptable mesh resolution, for more details see Online Resource.

In this work an adaptive timestep approach was used and bounded by the Courant-Friedrichs-Lewy (CFL) number, to reduce the computational cost. In all simulations the maximum CFL number was limited to 5, corresponding to a timestep size in the order of $160 \mu \mathrm{s}$ at peak flow and an average CFL number of $0.3-0.5$ which is within the recommend interval to limit artificial turbulence damping. ${ }^{1}$ Results from a temporal sensitivity analysis showed very small discrepancy between this approach and two more restrict timestep criterias, but with a threefold decrease of the computing cost.

The spatial and temporal discretizations were performed with second-order central difference and backward Euler scheme. All computations were converged to a root-mean-square residual target of $10^{-5}$. Simulations were run on the high performance cluster Triolith at the National Supercomputer Centre (NSC), Linköping, Sweden. On average 4.5 wallclock hours (650 CPU hours) per cardiac cycle was required per case, using 160 cores.

\section{Post-processing}

By applying the Reynolds decomposition on the raw velocity signal, the velocity fluctuations can be distinguished from the mean velocity components $(\bar{u}, \bar{v}$ and $\bar{w})$, described by the RMS difference between the instantaneous and mean velocity ${ }^{54}$ :

$$
u^{\prime}=\sqrt{\frac{1}{N} \sum_{i=1}^{N}\left(u_{i}-\bar{u}\right)^{2}}
$$

where $N$ is the number of samples. In a pulsatile dataset, $N$ would correspond to the number of available cardiac cycles, thus Eq. (1) represents a phase averaged quantity. The specific TKE (i.e., energy per given volume) is defined as the kinetic energy of the fluctuating velocity components according to:

$$
T K E=\frac{1}{2} \rho\left(u^{\prime 2}+v^{\prime 2}+w^{\prime 2}\right)
$$

where $\rho$ is the density and $u^{\prime}, v^{\prime}$ and $w^{\prime}$ are the fluctuating velocity components. Similarly, the specific kinetic energy (KE) of the mean velocity components is defined as:

$$
K E=\frac{1}{2} \rho\left(\bar{u}^{2}+\bar{v}^{2}+\bar{w}^{2}\right)
$$

To show the volumetric $T K E$ variation over the cardiac cycle, the specific $T K E$ was integrated in $3 \mathrm{D}$ space. To evaluate the total amount of $T K E$ over the entire cardiac cycle, both spatial and temporal integration were performed. In this work a minimum of 20 cardiac cycles was used to guarantee sufficient turbulence statistics (see Online Resource), excluding the initial cycle. Velocity data was collected every $0.01 \mathrm{~s}$.

In-plane flow eccentricity was quantified similarly to previous work assessing BAV flow jets, ${ }^{50}$ where the Euclidean distance between the geometrical center of the vessel and the center of velocity was used. In this work flow eccentricity was based on the displacement between the center of kinetic energy $\left(C_{\mathrm{KE}}\right)$ and the lumen centroid. At a given cross-sectional plane the $C_{\mathrm{KE}}$ is defined as:

$$
C_{\mathrm{KE}}=\frac{1 / n \sum_{i=0}^{n} r_{i} K E_{i}}{1 / n \sum_{i=0}^{n} K E_{i}}
$$

where $n$ is the total number in-plane pixels and $r$ the pixel position. The numerator calculates the $K E$ weighted average lumen pixel position, normalized by the mean $K E$ (denominator).

The spatiotemporal distribution of both TKE and flow eccentricity were computed at streamwise locations over the cardiac cycle. Both parameters were assessed in cross-sectional planes normal to the centerline with an incremental distance of $2 \mathrm{~mm}$. 


\section{RESULTS}

The trans-coarctation pressure gradient versus centerline position (Fig. 14 in Online Resource) revealed a maximum instantaneous drop in the order of $11 \mathrm{mmHg}$ before treatment, which is below the limit regarded as a significant $\mathrm{CoA}(>20 \mathrm{mmHg})^{4}$ but still a residual gradient that motivates further attention. As a result of the interventions, the pressure loss was reduced substantially for all cases.

The volumetric rendering of peak TKE at early systolic flow deceleration (Fig. 3) displayed distinct laminar-to-turbulence transition in the proximal part of the LCCA and kinking of the arc, independent of the distal morphology changes. As the flow exit the stenosis different turbulence levels could be noticed as a result of the interventions, in general indicating a more concentric and narrower extension of the most energetic part of the TKE field compared to baseline.
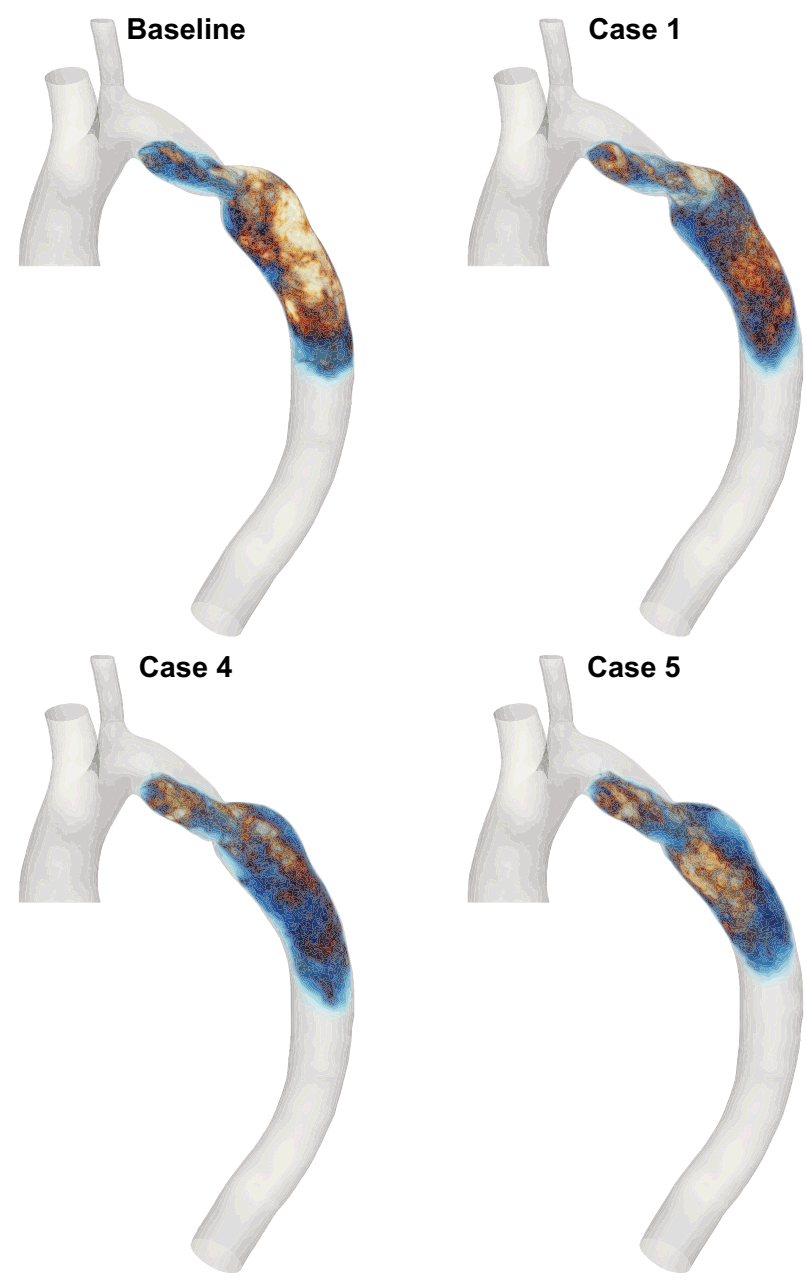

The integrated TKE over the post-stenotic volume (Fig. 4a) showed similar onset of turbulence (peak systole) and maximum intensity (beginning of flow deceleration), in line with previous knowledge that acceleration tends to stabilize the flow, while deceleration allows for transition to turbulence. ${ }^{46}$ Pre-intervention, a steep decaying gradient was observed as flow started to decelerate further. Post-intervention, the TKE-peak dropped followed by a slower decay throughout the systolic deceleration as a result of increased dilatation. At early-diastole, the loss of flow momentum rapidly reduced most of the prevailing turbulent flow among the cases. The total amount of TKE revealed an inverse relationship against the change in smallest cross-sectional area of the stenosis (Fig. 4b), where C6 showed more than a twofold reduction compared to baseline. However, most of this turbulence reduction occurred within the first $30 \%$ of stenotic dilatation (baseline to $\mathrm{C} 2$ ), where after the

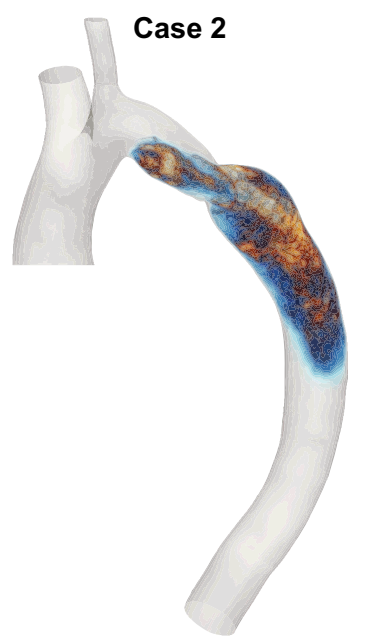

Case 6

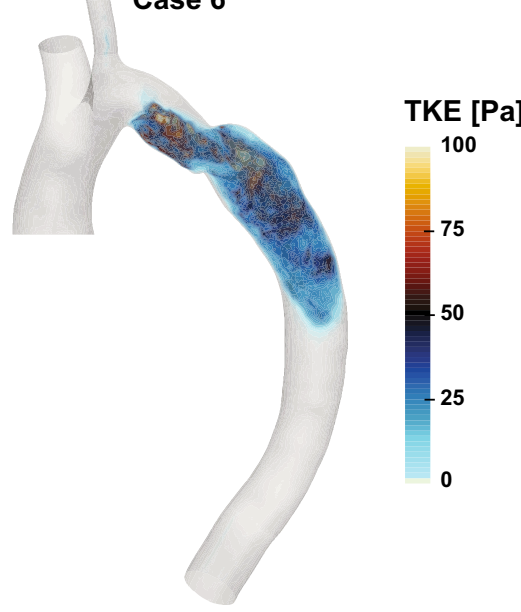

Case 3
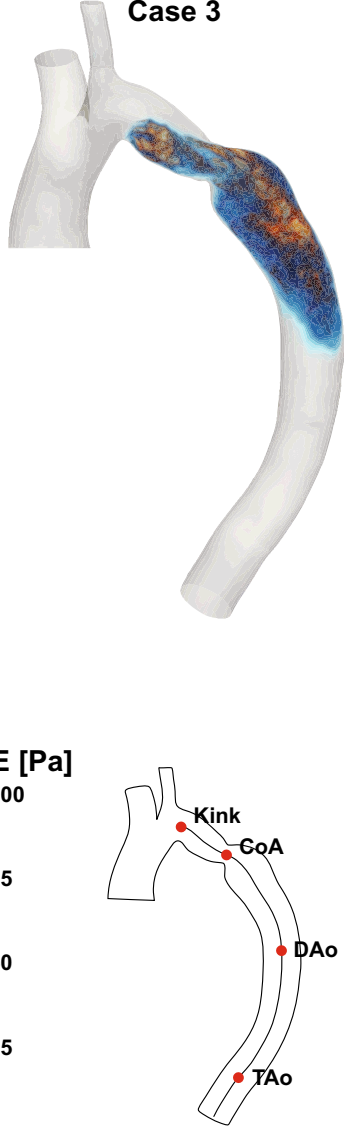

FIGURE 3. Volumetric rendering of turbulent kinetic energy (TKE), shown at corresponding maximum intensity in the early deceleration phase (Fig. 4a). In all cases, turbulence initiates at the Kink of the aortic arch and intensifies distal to the CoA. As a result of the interventions (Case 1-6) the flow will develop differently, where the most energetic part of the TKE overall show a more concentric and less distributed field compared to pre-treatment (Baseline). Note, for visualization purposes the colormap was thresholded at $100 \mathrm{~Pa}$. A time-resolved animation of the TKE for Baseline and Case 5 is given in Online Resource 2. 


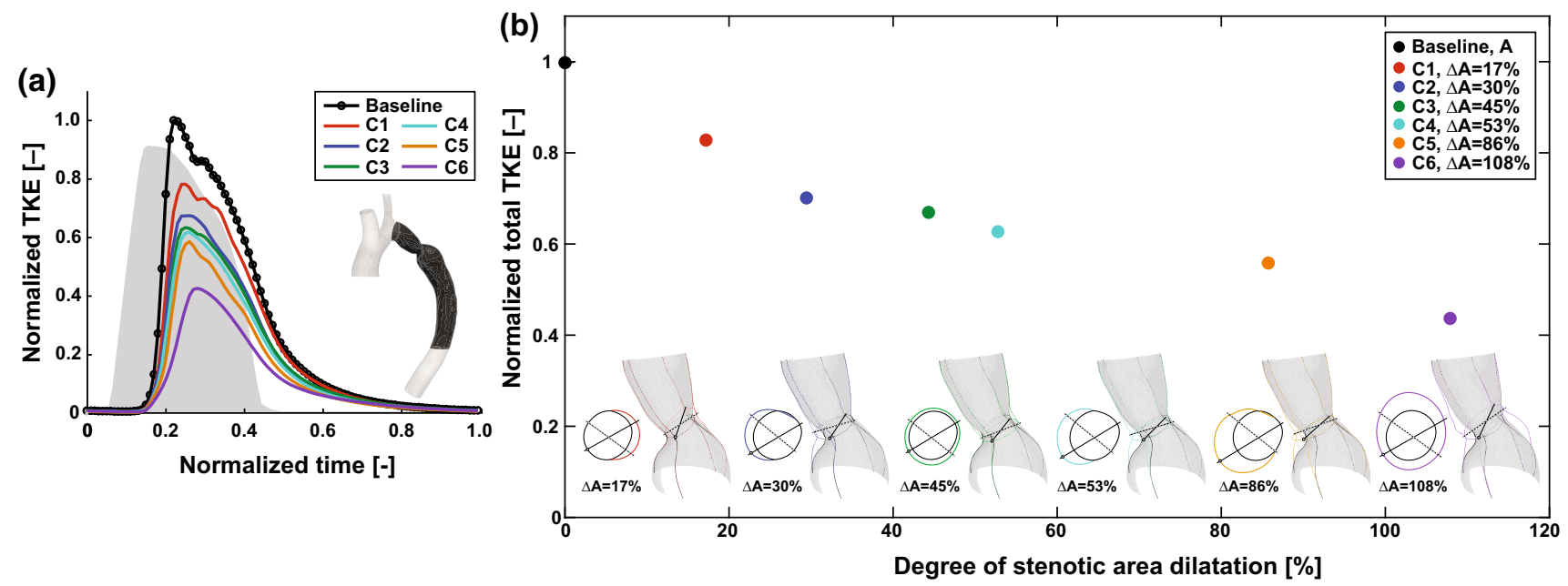

FIGURE 4. (a) Volume integrated turbulent kinetic energy (TKE) over the most pronounced turbulent region (highlighted in black in the vessel). Data were normalized with the pre-treated (Baseline) peak value. For comparison the input flow rate was included as a grey background. Notice that the transition to turbulence initiates at peak systole and that maximum TKE occurs during flow deceleration. (b) Total amount of TKE versus the degree of smallest stenotic cross-sectional area increase. Notice that the total TKE tends to level out in response to further dilatation of the coarctation. The total TKE was computed by temporal integration of the volume integrated TKE and thereafter normalized with Baseline.

total TKE showed a more asymptotic behavior in response to further CoA enlargement. Additionally, a linear relationship was found between the reductions of peak volume integrated TKE and the reduced level of total TKE (Fig. 5).

The spatiotemporal distribution of TKE pre-intervention (BL) revealed broad intensities distal the CoA, with the most elevated energy levels seen in the immediate post-stenotic region during the initial part of flow decceleration, extending to the DAo (Fig. 6). After intervention, an overall TKE reduction could be observed, in line with Fig. 4, where outer curvature dilatation $(\mathrm{Cl})$ have a tendency to maintain the spatial stretch further into the deceleration phase compared to inner curvature dilatations $(\mathrm{C} 2, \mathrm{C} 4-\mathrm{C} 5)$ where turbulence appeared to dissipate faster. For the intermediate intervention (C3) a mix of the above mentioned features could be depicted. The most dilated intervention (C6) showed the least amount of TKE intensity. In the vicinity of the $\mathrm{CoA}$, indications of relaminarization could be seen as a mild TKE drop, which was less pronounced as the degree of dilatation of the stenosis was increased.

The velocity magnitude at the center plane of the vessel showed a clear jet formation at the throat of the stenosis that partly rolled-up to secondary flow features, before colliding with the outer curvature wall at different intensities (Fig. 7). For the four least dilated interventions $(\mathrm{C} 1-\mathrm{C} 4)$, the corresponding jet direction and velocity intensity suggested continuous wall impingement. For $\mathrm{C} 5$, a more concentric and less energetic jet trajectory could be observed, resulting in a less severe wall impingement. For C6 on the other

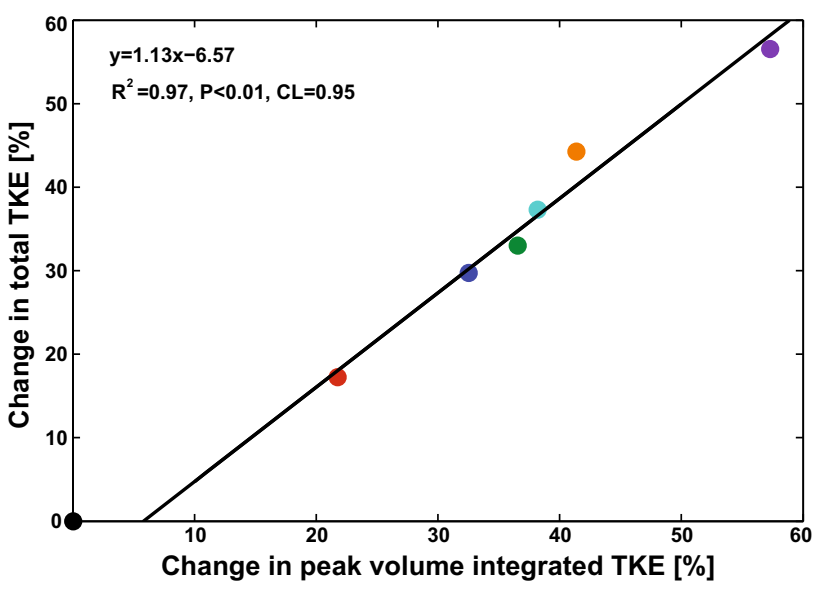

FIGURE 5. The change in peak volume integrated turbulent kinetic energy (TKE) ( $x$-axis) plotted against the change in total amount of TKE ( $y$-axis). That is, the reduction of instantaneous peak values at early flow deceleration versus the overall reduction integrated over the entire cardiac cycle, see Figs. $4 a$ and $4 b$ respectively. A linear regression was performed, with corresponding statistics. For legend, see Fig. 4b.

hand, the jet was directed more towards the outer wall, but despite this unfavorable direction most of the jet intensity was lost just before the wall. In fact, a somewhat similar jet direction could be seen between $\mathrm{C} 4$ and $\mathrm{C} 6$, but the stronger jet formation through the former $\mathrm{CoA}$ induced higher velocities at the opposing wall. From the detachment point of the jet to the distal segments, large zones of low intensity flows could be noticed in conjunction to more asymmetric flow conditions, which was less pronounced in C5 and C6.

Before intervention the flow eccentricity map showed an early onset of flow displacement just distal 

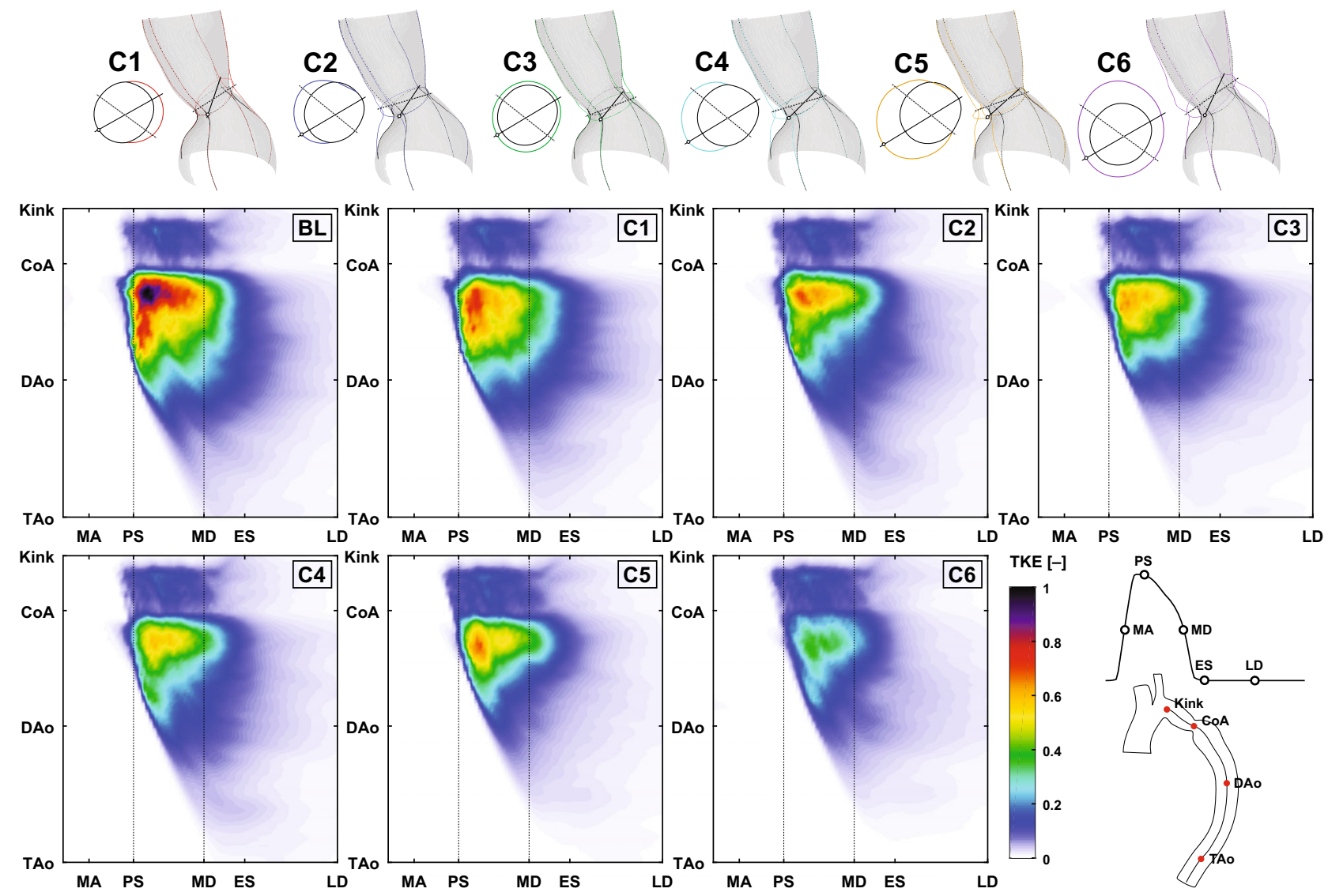

FIGURE 6. Spatiotemporal map of cross-sectional area integrated turbulent kinetic energy (TKE). Data was assessed at planes normal to the centerline with an incremental distance of $2 \mathrm{~mm}$, corresponding to $98 \times 75$ (slices $\times$ timesteps) data points. All cases were normalized with the Baseline (BL) peak values. $X$-axis labels are MA: maximum acceleration, PS: peak systole, MD: maximum deceleration, ES: end of systole, and LD: late diastole. $Y$-axis label positions are represented by the Baseline configuration (indicated in the lower right subplot), and are: Kink (the elbow of the aortic arch), CoA (coarctation of the aorta), DAo (descending aorta) and TAo (thoracic aorta).

to the CoA, stretching beyond the DAo during the deceleration phase before returning to the center of the lumen at the end of systole (Fig. 8, BL). Post-intervention, a general reduction of flow eccentricity could be observed among the cases, where $\mathrm{C} 4-\mathrm{C} 6$ showed the most concentric flow conditions in the jet dominant region (to the halfway point of the DAo). For the inner curvature dilated CoAs $(\mathrm{C} 2, \mathrm{C} 4-\mathrm{C} 5)$ this jet eccentricity was gradually reduced during flow acceleration, which later tended to enhance the flow displacement further downstream as the jet maintained more of its momentum compared to baseline (specifily noted in $\mathrm{C} 2$ vs. C4, see also Fig. 7). Between $\mathrm{C} 1$ and $\mathrm{C} 3$ a similar response could be depicted, but in contrast to the former cases the eccentricity of the jet were retained during a longer part of deceleration phase, lacking the distal enhancement. At peak systole, the centroid of flow eccentricity was predominately displaced towards the outer wall in all cases with the exception of C5 and
C6, where an overall alignment with the geometrical centerline could be observed (Fig. 9).

\section{DISCUSSION}

This study applied large eddy simulation to quantify the change in turbulent kinetic energy and flow eccentricity in a pre-treated coarctation and in a number of virtual follow-up intervention scenarios. Results showed an inverse asymptotic relationship between the total amount of TKE and degree of dilatation of the coarctation, where pre-stenotic geometry may limit the possible improvement by treating the CoA alone. Spatiotemporal maps of TKE and flow eccentricity could be coupled to the characteristics of the jet, suggesting that concentric dilatation of the CoA may not always be the ideal choice to restore normal flow conditions. 


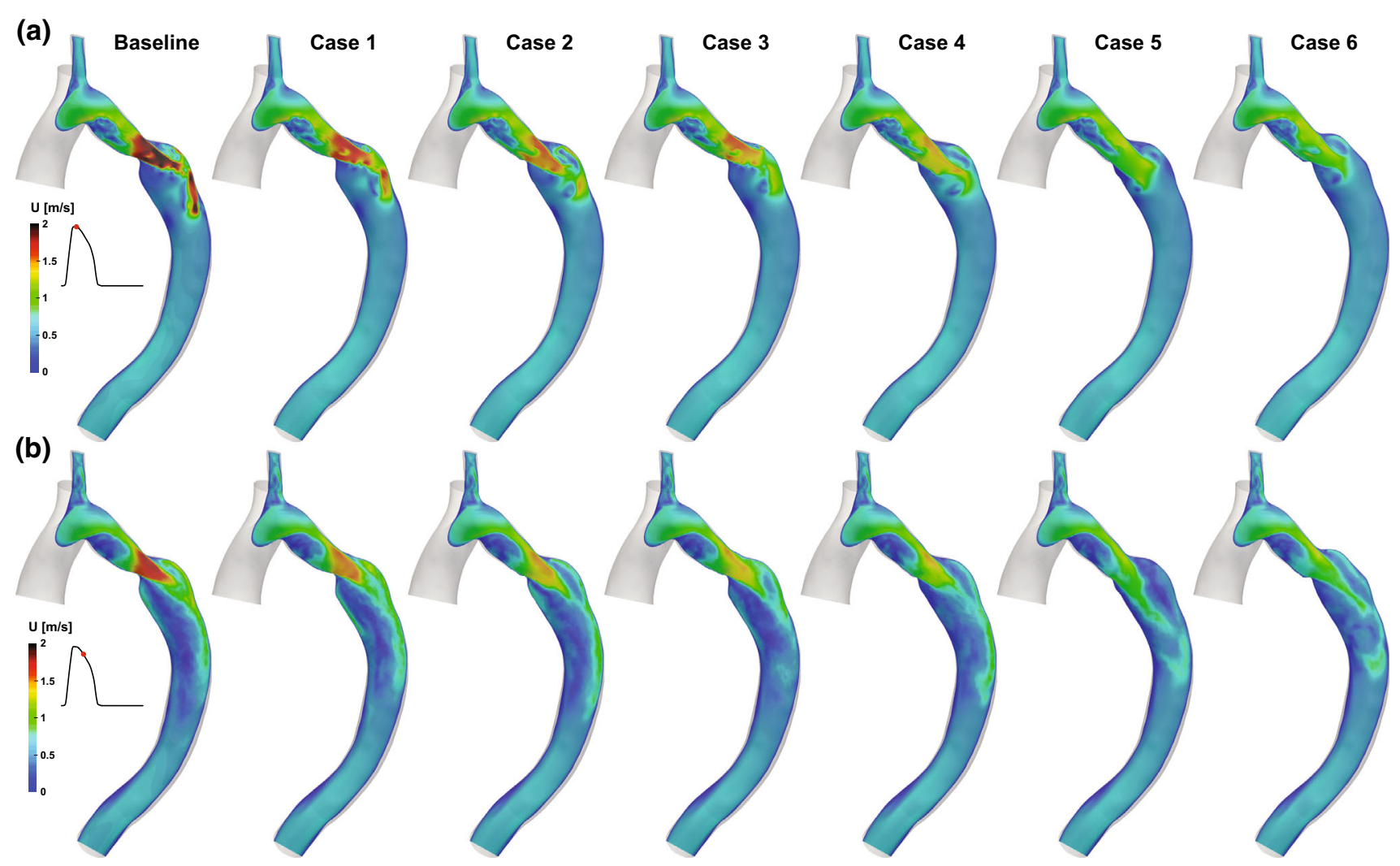

FIGURE 7. Velocity magnitude displayed on a cut-plane at the center of the vessels at (a) peak systole and (b) early systolic flow deceleration. For Baseline and in a majority of the interventions (Case 1-4) the jet direction and intensity indicates substantial wall impingement. For Case 5 and 6 , a more concentric and weaker jet can be seen, with reduced velocity intensity at the opposite wall. Also notice the large regions of low intensity flow as results of the different jet trajectories. A time-resolved animation of the centerplane velocity magnitude is given in Online Resource 3.

The volume integrated TKE showed similar characteristics as previously reported studies comparing CFD and MRI-derived TKE. ${ }^{32}$ In the work by Lantz et al., ${ }^{32}$ the volume integrated TKE was computed before and after intervention, showing peak intensities at early flow deceleration followed by a much steeper TKE decay for the pre-treated model. These findings are in line with the observations in this work and can also be confirmed in a recent experimental study of stenotic carotid models using particle image velocimetry. ${ }^{28}$

To account for the total amount TKE over the cardiac cycle, the volumetric integrated TKE was also integrated over time. Results showed an asymptotic relationship between the total TKE and stenosis area, revealing a less pronounced TKE reduction with increased dilatation. The asymptotic behavior can be explained by the upstream turbulence, initiated at the gothic shaped aortic arc, which is not fully relaminarized at the throat of the CoA. In fact, as the stenosis is further dilated, turbulent flow may remain from the upstream region and consequently contribute to the TKE levels in the post-stenotic region. Thus, only a certain amount of TKE reduction can be achieved by treating this coarctation alone. A turbulence response curve (such as Fig. 4b) could be used to e.g., determine to which extent the coarctation could be dilated in order to reach a certain satisfactory limit. Furthermore, the asymptotic level may also be used to indicate the significance of any upstream geometry composition effects on turbulence production, hence motivating a more comprehensive intervention procedure.

The strong relationship found between the changes in maximum volume integrated TKE and change in total TKE indicates that the overall reduction of TKE may be quantified by the peak value alone. Besides the benefit of handling a smaller amount of data clinically, excluding the time integration also reduced the accumulation of noise at the low turbulent intensity ranges and analysis time. Furthermore, as noted in Lantz et al., ${ }^{32}$ a steady LES captured peak integrated TKE very well when compared to a pulsatile LES at the same flow rate. Additionally, it has also been argued that the possible error induced by the SGS model would be less pronounced at peak flow conditions due to the more developed turbulence field. ${ }^{35}$ By only 

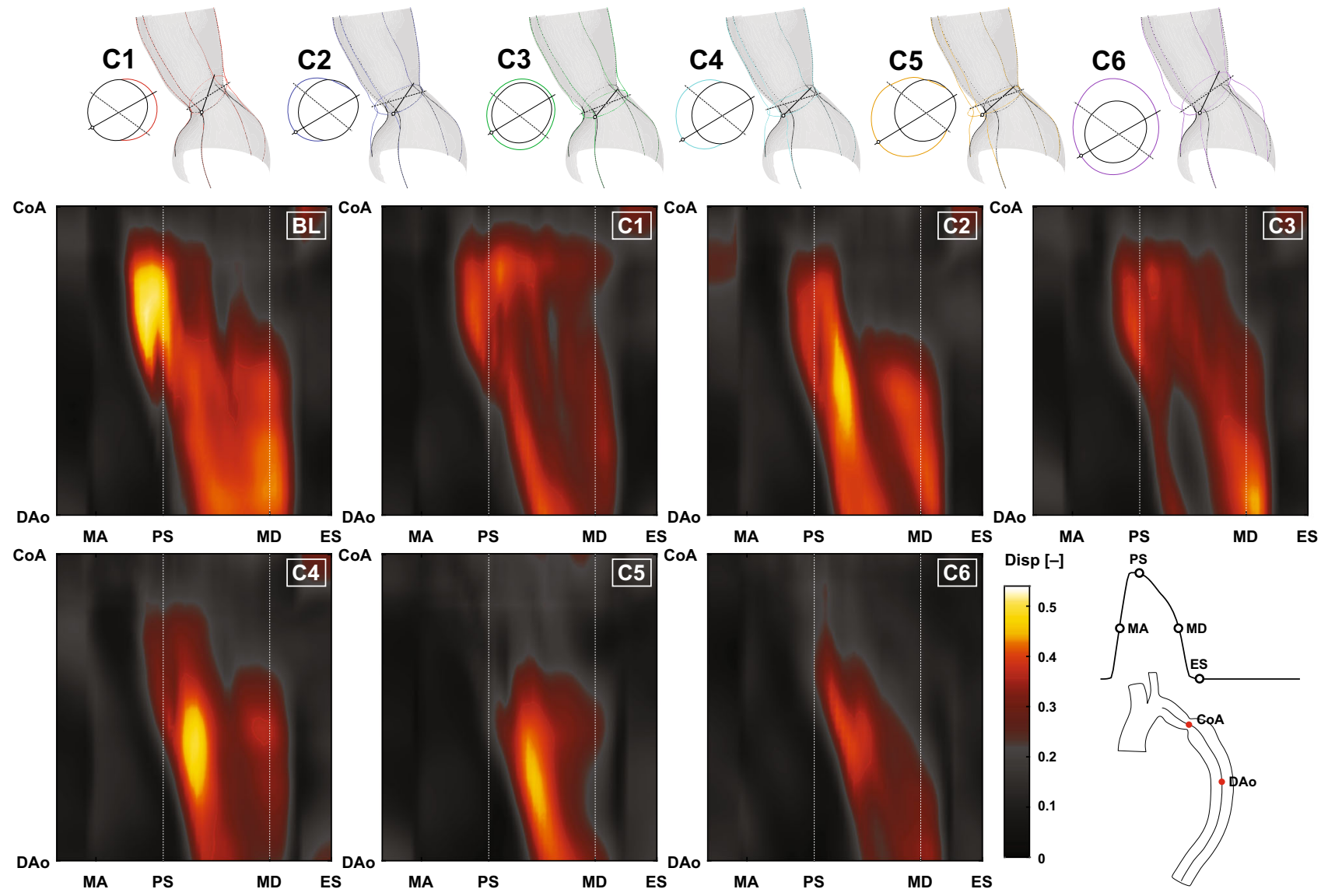

FIGURE 8. Spatiotemporal map of flow eccentricity. The displacement of the flow was assessed at planes normal to the centerline with an incremental distance of $2 \mathrm{~mm}$, corresponding to $37 \times 50$ (slices $\times$ timesteps) data points. Data was normalized with the mean local radius, where 0 indicates that the energy of the flow is predominantly aligned with the vessel center whereas a value of 1 indicates a flow that is completely displaced towards the wall. For axis legend, see Fig. 6.

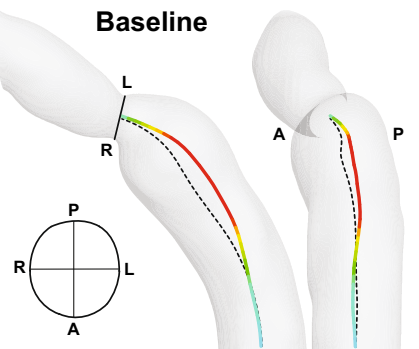

Case 4

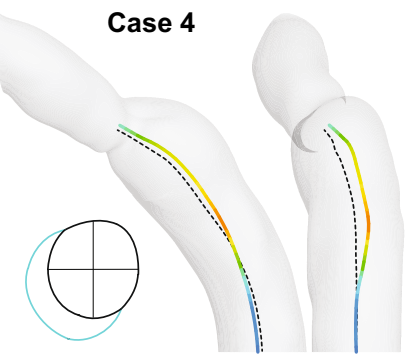

Case 1

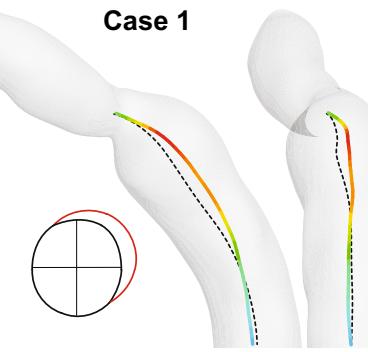

Case 5

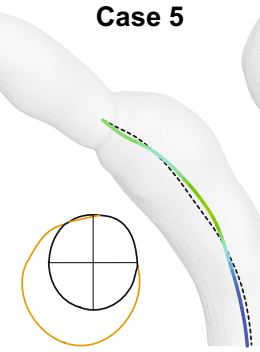

Case 2

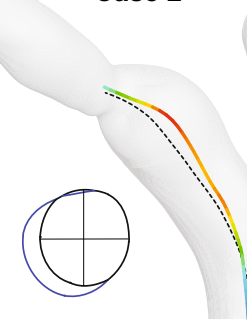

Case 6

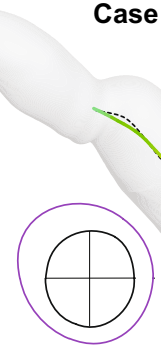

Case 3

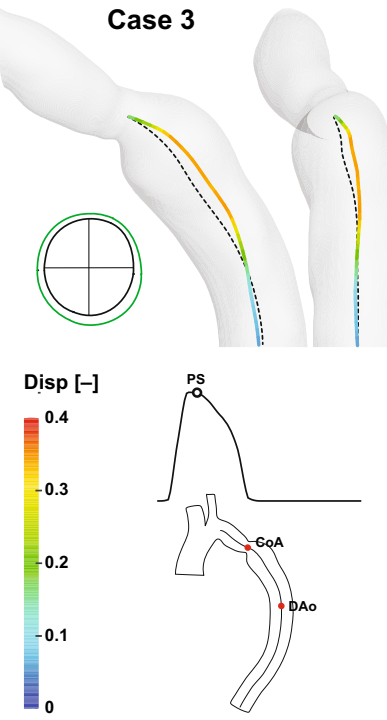

FIGURE 9. Flow eccentricity line versus the geometrical centerline (dashed-black) at peak systole (PS). The intensity can also be depicted via the vertical line in Fig. 8. All configurations, except for Case 5 and 6 , show predominated flow displacement towards the outer wall in the post-stenotic region, that latter return to a more centralized flow around the point of descending aorta (DAo). In Case 5 the flow is more aligned with the vessel center. Each case is highlighted in the left-right (L-R) and anterior-posterior (A-P) view of the aorta. 
simulating steady LES the computational cost could be reduced substantially compared to a pulsatile counterpart, where as much as 20 cardiac cycles have been proven necessary to obtain sufficient phase average results (see Online Resource). In a combined framework, accuracy and speed should be enhanced in order to approach clinical practice.

The spatiotemporal TKE maps could be linked to the observations in the eccentricity map, where the timing and strength of the post-stenotic jet shows to play a major role in the spread and intensity of turbulence. For this patient, outer curvature dilatation of the CoA seems to preserve the jet flow displacement longer into the cardiac phase, which also is reflected on the turbulence, whereas the inner curvature interventions tends to promote an overall more concentric jet (e.g., C3 vs. C4) that extends the turbulence further downstream. However, despite a favorable jet direction enough momentum can cause the jet to impinge on the opposite wall to a significant amount (e.g., in C4). If the distance to the wall, however, is large enough and the momentum of the jet is weaker, most of the jet will be broken down before the wall (e.g., in C5C6). These findings are consistent with previous work by Lantz et al., ${ }^{32}$ where a qualitative assessment of the jet direction pre and post CoA treatment indicated that a more concentric flow conditions enhance the distal transport of turbulence more efficiently, in spite of a substantially weaker jet and reduced amount of total TKE. In rabbits with CoA, unfavorable jet directions have shown elevated shear forces at the impingement zone of the wall, causing endothelial dysfunction and reduced smooth muscle contractility. ${ }^{38}$ In all cases severe post-stenotic dilation could be depicted. In the clinical study by Reijer et al., ${ }^{14}$ a significant correlation could be found between aortic dilation and BAV jet angle, suggesting that flow eccentricity has an important role in vascular remodeling. Eccentric flow conditions are also inherently accompanied with larger regions of low velocity intensity; regions which have been associated to plaque formation and thrombogenesis. $22,26,27,58$

In general, there are assumptions and limitations associated with patient-specific CFD simulations that may affect the results. This patient suffered from arterial hypertension, which has been associated to arterial stiffening. ${ }^{21,41}$ However, it is important to emphasize that the purpose of this study was to compare the overall flow characteristics between different intervention outcomes rather than aiming for absolute values. The rigid wall assumption may alter the flow field compared to a compliant model simulation, which could contribute to additional turbulence dissipation. Opposed to an inlet velocity plug, a complete timevarying velocity profile containing all velocity components, ${ }^{20}$ including aortic valve morphology, ${ }^{57}$ have shown to have an impact on the local hemodynamics. However, for this model the strong flow momentum initiated at the kink separation, the long downstream distance to the CoA, and the narrowing itself will likely dampen most the upstream flow details provided at the inlet. The virtual interventions in this study are examples of dilatations, and hence not intended to simulate one particular intervention procedure but rather investigating the potential outcome. Thus, the clinical challenges were not addressed in the present work. Nevertheless, an eccentric CoA dilatation may be plausible if the force distribution from a catheter balloon expansion could be controlled, e.g., by a tailormade balloon where the deformation rate is governed by the asymmetric properties of balloon wall. The eccentric/concentric counter-part for the same degree of dilatation was not investigated in this study. However, in the experimental work by Kefayati et al. ${ }^{28}$ the eccentric stenoses did not favor TKE reduction to the same degree as its concentric counter-parts.

This numerical intervention study, of a patientspecific coarctation, has shown the geometrical impact on turbulence levels and flow eccentricity. New insights revealed that for this patient an asymptotic decay of TKE could be observed in response to more CoA enlargement, with the most distinct turbulence reduction at the lowest degrees of stenotic dilatation. These findings suggest that the interventions may be limited by the pre-stenotic hypoplastic segments and if further improvement is motivated, a larger portion of the vessel needs to be treated. Results further indicated that the properties of the post-stenotic flow eccentricity plays an important role in the distal turbulence response, where the level of flow displacement and duration of the jet influence the spread and exposure time of the distal TKE field. The total TKE can give an indication of the amount of turbulent flow and the spatiotemporal map of flow eccentricity is a comprehensive way of evaluating the asymmetry of the flow, representing two measurable quantities that could assess CoA severity via MRI measurements. With CFD simulations an intervention response-map could be established, which could also include non-measurable hemodynamic metrics such as WSS, to better predict the post-treatment outcome. In a combined MRI-CFD framework, predictions have the possibility to be validated at any therapeutic stage, providing a platform for improving CoA therapy on a patient-specific level. 


\section{ELECTRONIC SUPPLEMENTARY MATERIAL}

The online version of this article (doi: 10.1007/s13239-015-0243-9) contains supplementary material, which is available to authorized users.

\section{ACKNOWLEDGEMENTS}

This research was supported by grants from the Swedish Research Council and the Center for Industrial Information Technology (CENIIT). We also like to acknowledge the Center of Medical Image Science and Visualization (CMIV, http://www.cmiv.liu.se/) for providing necessary MRI data and the National Supercomputer Centre (NSC) for the computational resources via grants from the Swedish National Infrastructure for Computing (SNIC).

\section{CONFLICT OF INTEREST}

M. Karlsson received non-financial support from the Swedish National Infrastructure for Computing, T. Ebbers grants from the Center for Industrial Information Technology at Linköping University, the Swedish Research Council and the Swedish e-Science Research Center.

\section{STATEMENT OF HUMAN STUDIES}

All procedures followed were in accordance with the ethical standards of the responsible committee on human experimentation (institutional and national) and with the Helsinki Declaration of 1975 , as revised in 2000 (5). Informed consent was obtained from all patients for being included in the study.

\section{STATEMENT OF ANIMAL STUDIES}

No animal studies were carried out by the authors for this article.

\section{REFERENCES}

${ }^{1}$ Ansys Inc. ANSYS CFX-Solver Modeling, Academic Research, Release 14.0. Ansys Inc.

${ }^{2}$ Arzani, A., P. Dyverfeldt, T. Ebbers, and S. C. Shadden. In vivo validation of numerical prediction for turbulence intensity in an aortic coarctation. Ann. Biomed. Eng. 4:860 870, 2012.

${ }^{3}$ Barker, A. J., M. Markl, J. Bürk, R. Lorenz, J. Bock, S. Bauer, J. Schulz-Menger, and F. von KnobelsdorffBrenkenhoff. Bicuspid aortic valve is associated with al- tered wall shear stress in the ascending aorta. Circ. Cardiovasc. Imaging 4:457-466, 2012.

${ }^{4}$ Baumgartner, H., P. Bonhoeffer, N. M. De Groot, F. de Haan, J. E. Deanfield, N. Galie, M. A. Gatzoulis, C. Gohlke-Baerwolf, H. Kaemmerer, P. Kilner, F. Meijboom, B. J. Mulder, E. Oechslin, J. M. Oliver, A. Serraf, A. Szatmari, E. Thaulow, P. R. Vouhe, E. Walma, and Task Force on the Management of Grown-up Congenital Heart Disease of the European Society of Cardiology (ESC), Association for European Paediatric Cardiology (AEPC), ESC Committee for Practice Guidelines (CPG). ESC Guidelines for the management of grown-up congenital heart disease (new version 2010). Eur. Heart J. 23:29152957, 2010.

${ }^{5}$ Binter, C., R. Manka, S. H. Sündermann, V. Knobloch, M. Stuber, and S. Kozerke. Assessment of energy loss in aortic stenosis using Bayesian multipoint phase-contrast MRI. J. Cardiovasc. Magn. Reson. 15(Suppl 1):P47, 2013.

${ }^{6}$ Caballero, A., and S. Laín. A review on computational fluid dynamics modelling in human thoracic aorta. Cardiovasc. Eng. Technol. 2:103-130, 2013.

${ }^{7}$ Carreau, P. J. Rheological equations from molecular network theories (1957-1977). Trans. Soc. Rheol. 1:99-127, 1972.

${ }^{8}$ Cho, Y., and K. Kensey. Effects of the non-Newtonian viscosity of blood on flows in a diseased arterial vessel. Part 1: steady flows. Biorheology 3-4:241-262, 1990.

${ }^{9}$ Cohen, M., V. Fuster, P. Steele, D. Driscoll, and D. McGoon. Coarctation of the aorta. Long-term follow-up and prediction of outcome after surgical correction. Circulation 4:840-845, 1989.

${ }^{10}$ Coogan, J. S., F. P. Chan, J. F. LaDisa, Jr, C. A. Taylor, F. L. Hanley, and J. A. Feinstein. Computational fluid dynamic simulations for determination of ventricular workload in aortic arch obstructions. J. Thorac. Cardiovasc. Surg. 2:489-495, 2013.

${ }^{11}$ Coogan, J. S., F. P. Chan, C. A. Taylor, and J. A. Feinstein. Computational fluid dynamic simulations of aortic coarctation comparing the effects of surgical-and stentbased treatments on aortic compliance and ventricular workload. Catheter Cardiovasc. Interv. 5:680-691, 2011.

${ }^{12}$ Coogan, J. S., J. D. Humphrey, and C. A. Figueroa. Computational simulations of hemodynamic changes within thoracic, coronary, and cerebral arteries following early wall remodeling in response to distal aortic coarctation. Biomech. Model. Mechanobiol. 1:79-93, 2013.

${ }^{13}$ Davidson, L. Large eddy simulations: how to evaluate resolution. Int. J. Heat Fluid Flow 5:1016-1025, 2009.

${ }^{14}$ den Reijer, P. M., D. Sallee, III, P. van der Velden, E. R. Zaaijer, W. J. Parks, S. Ramamurthy, T. Q. Robbie, G. Donati, C. Lamphier, and R. P. Beekman. Hemodynamic predictors of aortic dilatation in bicuspid aortic valve by velocity-encoded cardiovascular magnetic resonance. $J$. Cardiovasc. Magn. Reson. 12:4, 2010.

${ }^{15}$ Dyverfeldt, P., M. D. Hope, E. E. Tseng, and D. Saloner. Magnetic resonance measurement of turbulent kinetic energy for the estimation of irreversible pressure loss in aortic stenosis. JACC Cardiovasc. Imaging 1:64-71, 2013.

${ }^{16}$ Dyverfeldt, P., J. P. E. Kvitting, A. Sigfridsson, J. Engvall, A. F. Bolger, and T. Ebbers. Assessment of fluctuating velocities in disturbed cardiovascular blood flow: in vivo feasibility of generalized phase-contrast MRI. J. Magn. Reson. Imaging 3:655-663, 2008.

${ }^{17}$ Faggiano, E., L. Antiga, G. Puppini, A. Quarteroni, G. B. Luciani, and C. Vergara. Helical flows and asymmetry of 
blood jet in dilated ascending aorta with normally functioning bicuspid valve. Biomech. Model. Mechanobiol. 4:801-813, 2013.

${ }^{18}$ Gårdhagen, R., F. Carlsson, and M. Karlsson. Large Eddy simulation of pulsating flow before and after CoA repair: CFD for intervention planning. Adv. Mech. Eng. 7:971418, 2015.

${ }^{19}$ Gårdhagen, R., J. Lantz, F. Carlsson, and M. Karlsson. Large Eddy simulation of stenotic flow for wall shear stress estimation-validation and application. WSEAS Trans. Biol. Biomed. 3:86-101, 2011.

${ }^{20}$ Goubergrits, L., R. Mevert, P. Yevtushenko, J. Schaller, U. Kertzscher, S. Meier, S. Schubert, E. Riesenkampff, and T. Kuehne. The impact of MRI-based inflow for the hemodynamic evaluation of aortic coarctation. Ann. Biomed. Eng. 12:2575-2587, 2013.

${ }^{21}$ Hager, A., S. Kanz, H. Kaemmerer, C. Schreiber, and J. Hess. Coarctation Long-term Assessment (COALA): significance of arterial hypertension in a cohort of 404 patients up to 27 years after surgical repair of isolated coarctation of the aorta, even in the absence of restenosis and prosthetic material. J. Thorac. Cardiovasc. Surg. 3:738-745, 2007.

${ }^{22}$ Hathcock, J. J. Flow effects on coagulation and thrombosis. Arterioscler. Thromb. Vasc. Biol. 8:1729-1737, 2006.

${ }^{23}$ Heiberg, E., J. Sjogren, M. Ugander, M. Carlsson, H. Engblom, and H. Arheden. Design and validation of segment-freely available software for cardiovascular image analysis. BMC Med. Imaging 10:1, 2010.

${ }^{24}$ Hope, M. D., T. Sedlic, and P. Dyverfeldt. Cardiothoracic magnetic resonance flow imaging. J. Thorac. Imaging 4:217-230, 2013

${ }^{25}$ Hope, M. D., J. Wrenn, M. Sigovan, E. Foster, E. E. Tseng, and D. Saloner. Imaging biomarkers of aortic disease: increased growth rates with eccentric systolic flow. $J$. Am. Coll. Cardiol. 4:356-357, 2012.

${ }^{26}$ Jackson, S. P., W. S. Nesbitt, and E. Westein. Dynamics of platelet thrombus formation. J. Thromb. Haemost. 7:17-20, 2009.

${ }^{27}$ Karino, T., and H. Goldsmith. Role of blood cell-wall interactions in thrombogenesis and atherogenesis: a microrheological study. Biorheology 4:587-601, 1983.

${ }^{28}$ Kefayati, S., D. W. Holdsworth, and T. L. Poepping. Turbulence intensity measurements using particle image velocimetry in diseased carotid artery models: effect of stenosis severity, plaque eccentricity, and ulceration. $J$. Biomech. 1:253-263, 2014.

${ }^{29}$ Kim, H. J., I. E. Vignon-Clementel, C. A. Figueroa, J. LaDisa, K. E. Jansen, J. A. Feinstein, and C. A. Taylor. On coupling a lumped parameter heart model and a three-dimensional finite element aorta model. Ann. Biomed. Eng. 11:2153-2169, 2009.

${ }^{30}$ Kwon, S., J. A. Feinstein, R. J. Dholakia, and J. F. LaDisa, Jr. Quantification of local hemodynamic alterations caused by virtual implantation of three commercially available stents for the treatment of aortic coarctation. Pediatr. Cardiol. 35:732-740, 2014

${ }^{31}$ Ladisa, J. F., C. A. Figueroa, I. E. Vignon-Clementel, H. J. Kim, N. Xiao, L. M. Ellwein, F. P. Chan, J. A. Feinstein, and C. A. Taylor. Computational simulations for aortic coarctation: representative results from a sampling of patients. J. Biomech. Eng. 9:091008, 2011.

${ }^{32}$ Lantz, J., T. Ebbers, J. Engvall, and M. Karlsson. Numerical and experimental assessment of turbulent ki- netic energy in an aortic coarctation. J. Biomech. 11:18511858, 2013.

${ }^{33}$ Lantz, J., R. Gårdhagen, and M. Karlsson. Quantifying turbulent wall shear stress in a subject specific human aorta using large eddy simulation. Med. Eng. Phys. 8:1139-1148, 2012.

${ }^{34}$ Lantz, J., and M. Karlsson. Large eddy simulation of LDL surface concentration in a subject specific human aorta. $J$. Biomech. 3:537-542, 2011.

${ }^{35}$ Liu, J., S. Chu, and P. Lu. Turbulence characteristics downstream of bileaflet aortic valve prostheses. J. Biomech. Eng. 2:118-124, 2000.

${ }^{36}$ Markl, M., P. J. Kilner, and T. Ebbers. Comprehensive 4D velocity mapping of the heart and great vessels by cardiovascular magnetic resonance. J. Cardiovasc. Magn. Reson. 7:1-22, 2011 .

${ }^{37}$ Marrero, V. L., J. A. Tichy, O. Sahni, and K. E. Jansen. Numerical study of purely viscous non-Newtonian flow in an abdominal aortic aneurysm. J. Biomech. Eng. 10:101001, 2014.

${ }^{38}$ Menon, A., D. C. Wendell, H. Wang, T. J. Eddinger, J. M. Toth, R. J. Dholakia, P. M. Larsen, E. S. Jensen, and J. F. LaDisa, Jr. A coupled experimental and computational approach to quantify deleterious hemodynamics, vascular alterations, and mechanisms of long-term morbidity in response to aortic coarctation. J. Pharmacol. Toxicol. Methods 1:18-28, 2012.

${ }^{39}$ Mittal, R., S. Simmons, and F. Najjar. Numerical study of pulsatile flow in a constricted channel. J. Fluid Mech. 485:337-378, 2003.

${ }^{40}$ Molla, M. M., and M. C. Paul. LES of non-Newtonian physiological blood flow in a model of arterial stenosis. Med. Eng. Phys. 8:1079-1087, 2012.

${ }^{41}$ Nichols, W., M. O'Rourke, and C. Vlachopoulos. McDonald's Blood Flow in Arteries: Theoretical, Experimental and Clinical Principles. New York: CRC Press, pp. 52-54, 2011.

${ }^{42}$ Nicoud, F., and F. Ducros. Subgrid-scale stress modelling based on the square of the velocity gradient tensor. Flow Turbul. Combust. 3:183-200, 1999.

${ }^{43} \mathrm{O}$ 'Rourke, M. F., and T. B. Cartmill. Influence of aortic coarctation on pulsatile hemodynamics in the proximal aorta. Circulation 2:281-292, 1971.

${ }^{44} \mathrm{Ou}$, P., E. Mousseaux, D. S. Celermajer, E. Pedroni, P. Vouhe, D. Sidi, and D. Bonnet. Aortic arch shape deformation after coarctation surgery: effect on blood pressure response. J. Thorac. Cardiovasc. Surg. 5:1105-1111, 2006.

${ }^{45}$ Paul, M. C., M. Mamunmolla, and G. Roditi. Large-Eddy simulation of pulsatile blood flow. Med. Eng. Phys. 1:153159, 2009

${ }^{46}$ Peacock, J., T. Jones, C. Tock, and R. Lutz. The onset of turbulence in physiological pulsatile flow in a straight tube. Exp. Fluids 1:1-9, 1998

${ }^{47}$ Rosenthal, E. Coarctation of the aorta from fetus to adult: curable condition or life long disease process? Heart 11:1495-1502, 2005.

${ }^{48}$ Ryval, J., D. Steinman, and A. Straatman. Two-equation turbulence modeling of pulsatile flow in a stenosed tube. $J$. Biomech. Eng. 5:625-635, 2004.

${ }^{49}$ Scotti, A., and U. Piomelli. Turbulence models in pulsating flows. AIAA J. 3:537-544, 2002.

${ }^{50}$ Sigovan, M., M. D. Hope, P. Dyverfeldt, and D. Saloner. Comparison of four-dimensional flow parameters for quantification of flow eccentricity in the ascending aorta. $J$. Magn. Reson. Imaging 5:1226-1230, 2011. 
${ }^{51}$ Tennekes, H., and J. L. Lumley. A First Course in Turbulence. Cambridge: MIT press, 1972.

${ }^{52}$ Toro-Salazar, O. H., J. Steinberger, W. Thomas, A. P. Rocchini, B. Carpenter, and J. H. Moller. Long-term follow-up of patients after coarctation of the aorta repair. Am. J. Cardiol. 5:541-547, 2002.

${ }^{53}$ Varghese, S. S., S. H. Frankel, and P. F. Fischer. Direct numerical simulation of stenotic flows. Part 2. Pulsatile flow. J. Fluid Mech. 582:281-318, 2007.

${ }^{54}$ Versteeg, H. K., and W. Malalasekera. An Introduction to Computational Fluid Dynamics: The Finite Volume Method. Harlow: Pearson Education, pp. 49-50, 2007.

${ }^{55}$ Viscardi, F., C. Vergara, L. Antiga, S. Merelli, A. Veneziani, G. Puppini, G. Faggian, A. Mazzucco, and G. B. Luciani. Comparative finite element model analysis of ascending aortic flow in bicuspid and tricuspid aortic valve. Artif. Organs 12:1114-1120, 2010.

${ }^{56}$ Vriend, J. W., and B. J. Mulder. Late complications in patients after repair of aortic coarctation: implications for management. Int. J. Cardiol. 3:399-406, 2005.

${ }^{57}$ Wendell, D. C., M. M. Samyn, J. R. Cava, L. M. Ellwein, M. M. Krolikowski, K. L. Gandy, A. N. Pelech, S. C.
Shadden, and J. F. LaDisa, Jr. Including aortic valve morphology in computational fluid dynamics simulations: initial findings and application to aortic coarctation. Med. Eng. Phys. 6:723-735, 2013.

${ }^{58}$ Wentzel, J. J., R. Corti, Z. A. Fayad, P. Wisdom, F. Macaluso, M. O. Winkelman, V. Fuster, and J. J. Badimon. Does shear stress modulate both plaque progression and regression in the thoracic aorta? Human study using serial magnetic resonance imaging. J. Am. Coll. Cardiol. 6:846854, 2005.

${ }^{59}$ Yoganathan, A. P., K. Chandran, and F. Sotiropoulos. Flow in prosthetic heart valves: state-of-the-art and future directions. Ann. Biomed. Eng. 12:1689-1694, 2005.

${ }^{60}$ Zajac, J., J. Eriksson, P. Dyverfeldt, A. F. Bolger, T. Ebbers, and C. Carlhäll. Turbulent kinetic energy in normal and myopathic left ventricles. J. Magn. Reson. Imaging 41(4):1021-1029, 2015.

${ }^{61}$ Zamir, M., P. Sinclair, and T. H. Wonnacott. Relation between diameter and flow in major branches of the arch of the aorta. J. Biomech. 11:1303-1310, 1992. 